\title{
REXS contribution to electronic ordering investigation in solids
}

T.A.W. Beale ${ }^{1}$, G. Beutier ${ }^{2}$, S.R. Bland ${ }^{1}$, A. Bombardi ${ }^{3}$, L. Bouchenoire ${ }^{4,5}$, O. Bunău ${ }^{6}$, S. Di Matteo ${ }^{7}$, J. Fernández-Rodríguez ${ }^{8}$, J.E. Hamann-Borrero ${ }^{9}$, J. Herrero-Martín ${ }^{10}$, V.L.R. Jacques ${ }^{11}$, R.D. Johnson ${ }^{12}$, A. Juhin ${ }^{13}$, T. Matsumura ${ }^{14}$, C. Mazzoli ${ }^{15,11, a}$, A.M. Mulders ${ }^{16}$, H. Nakao ${ }^{17}$, J. Okamoto ${ }^{17}$, S. Partzsch ${ }^{9}$,

A.J. Princep ${ }^{16}$, V. Scagnoli ${ }^{18}$, J. Strempfer ${ }^{19}$, C. Vecchini ${ }^{3}$, Y. Wakabayashi ${ }^{20}$, H.C. Walker ${ }^{19}$, D. Wermeille ${ }^{4,5}$, and Y. Yamasaki ${ }^{17}$

1 Department of Physics, University of Durham, Rochester Building, South Road, Durham DH1 3LE, UK

2 SIMaP, Grenoble-INP-CNRS-UJF, BP. 75, 38402 Saint-Martin d'Hères Cedex, France

3 Diamond Light Source Ltd., Rutherford Appleton Laboratory, Didcot OX11 0DE, UK

4 Department of Physics, University of Liverpool, Liverpool, L69 7ZE, UK

5 XMaS CRG Beamline (BM28), European Synchrotron Radiation Facility, BP. 220, 38043 Grenoble Cedex, France

${ }^{6}$ IMPMC, CNRS - Univ. Pierre et Marie Curie Paris VI, 4 place Jussieu, 75252 Paris, France

7 Équipe de Physique des Surfaces et Interfaces, Institut de Physique de Rennes, UMR CNRS-UR1 6251, Université de Rennes 1, 35042 Rennes Cedex, France

8 Advanced Photon Source, Argonne National Laboratory, Argonne, Illinois 60439, USA

9 Leibniz Institute for Solid State and Materials Research IFW Dresden, Helmholtzstrasse 20, 01069 Dresden, Germany

10 Institut de Ciència de Materials de Barcelona, CSIC, Campus Universitari de Bellaterra, 08193 Bellaterra, Barcelona, Spain

11 European Synchrotron Radiation Facility, 6 rue Jules Horowitz, BP. 220, 38043 Grenoble Cedex, France

12 Clarendon Laboratory, Department of Physics, University of Oxford, Oxford, OX1 3PU, UK

13 Institut de Minéralogie et de Physique des Milieux Condensés (IMPMC), Université Pierre et Marie Curie, CNRS, 4 place Jussieu, 75252 Paris Cedex 5, France

14 Department of Quantum Matter, AdSM, Hiroshima University, Higashi-Hiroshima, 739-8530, Japan

15 CNISM - Dipartimento di Fisica, Politecnico di Milano, p.le Leonardo Da Vinci 32, 20133 Milano, Italy

16 School of PEMS, UNSW, Canberra, ACT, 2600, Australia

17 Condensed Matter Research Center and Photon Factory, Institute of Materials Structure Science, High Energy Accelerator Research Organization, Tsukuba, Ibaraki 305-0801, Japan

18 Swiss Light Source, Paul Scherrer Institut, 5232 Villigen PSI, Switzerland

19 Deutsches Elektronen-Synchrotron (Hasylab at DESY), 22607 Hamburg, Germany

20 Division of Materials Physics, Graduate School of Engineering Science, Osaka University, Toyonaka 560-8531, Japan

\footnotetext{
${ }^{a}$ e-mail: claudio.mazzoli@polimi.it
} 
Received 5 March 2012 / Received in final form 23 March 2012

Published online 15 June 2012

Abstract. Resonant Elastic X-Ray Scattering (REXS) has played a fundamental role in understanding electronic properties and in revealing hidden order, local symmetries and exotic states realized in correlated solids. This article reports on some of the relevant scientific contributions and technical advances over the last 20 years, by providing a list of related publications produced by various groups all around the world. The given perspective is that of a group of young scientists involved at various times in the investigation of the beauty of electronic ordering by the REXS technique.

\section{Introduction}

A number of interesting physical phenomena are related to the quantum nature of the constituent particles building up a given system. In solid state physics electrons are the main actors, thus naturally their degrees of freedom have to be considered in detail. This is particularly the case when strong electronic correlations (in the broadest possible sense) are present, as in colossal magnetoresistance, non conventional and high temperature superconductivity, heavy fermion physics, multiferroicity and magneto-electricity. Spatial confinement at mesoscopic scales injects further complexity, modulating properties and possibly providing a handle to control interactions. In the last 20 years, a significant part of the solid state community has consistently invested a great effort to understand this kind of systems: the challenges in understanding the underlying physics and the possibilities offered in device realization have focused the investigation towards the electronic orderings (EO) involved, i.e. charge, spin and spatial arrangement. Together with mutual interactions, they constitute the key ingredients to understand the microscopic origin of the above phenomena.

The need to identify and understand the mechanisms at play has pushed the search for the most suitable investigation techniques. In this respect, REXS has shown the ability to provide unique information on the electronic valence states. In particular symmetries, structures and order parameter (hidden or high multipolar) interplay have all been successfully investigated. Rarely have all of these been addressed by other techniques.

In the following, the investigation of charge, spin, multipolar ordering and their co-action in specific classes of materials is reported. Obviously, the cited articles are just a fraction of the massive work produced over the years. As a consequence, the list provided is absolutely not exhaustive nor comprehensive. On the contrary, authors have just tried to collect citations to REXS papers recognized as important contributions in unraveling electronic ingredients of wide interest. Although part of the reported literature was proved to be inaccurate, especially the one related to signal interpretation, it was still important to the discussion and the progression of EO understanding. Moreover it should serve as a reminder for currently proposed (and some times still controversial) interpretations of $\mathrm{EO}$ related phenomena. By providing some relevant examples showing the power and beauty of REXS technique in relation to EO investigation, the authors aim to enlarge and strengthen their community: we hope this paper will help students and scientists new to the field in getting closer to it.

\section{Solid state REXS}

As extensively discussed by other authors in these same special topic issue [1,2], resonant X-ray techniques provides a core-hole spectroscopy point of view on matter. This 
immediately implies synchrotron specificity, due to the need to tune the impinging radiation (photon-in) energy to excite an electronic inner shell resonance. The characteristic binding energy of electrons in atoms (http://xdb.lbl.gov) naturally brings in ion selectivity. Moreover, the energy resolution currently achieved in the X-ray regime allows for fine band details investigation: often information coming from close lying electronic states can be resolved.

While pure absorption (as in XANES, for example) is constrained to zero momentum transfer $(\mathrm{Q}=0)$, scattering techniques (photon-out) do not suffer from this limitation. By exploiting the Q dependence, important information on EO real space properties can be retrieved: for example resonant reflectivity and diffraction measurements have proved to be essential in investigating electronic states squeezed at surfaces and interfaces, evidencing the importance of EO reconstructions in relation to the surface properties [3-11].

If the interest is focused on long correlated components of EO in solids, then diffraction can be exploited to enhance the usually very weak scattering signal. When diffraction is performed close to an adsorption edge of one of the ionic species constituting the crystal, then the literature often refers to Resonant X-ray Diffraction (RXD). The rest of this paper mainly deals with results obtained in the above regime.

X-ray diffraction probes crystals via the coherent sum of radiation-matter interaction events, modulated by Bragg phases. Intensity versus Q, polarization and azimuth dependences of the scattered photons encode information on the scattering source process as well as on the local and translational symmetry of matter, which may result in site selectivity and sensitivity to supermodulations (of suitable propagation vector). The average electronic structure can be determined, in relation to both charge and magnetism, as proved by some non resonant pioneering investigations performed on laboratory sources [12-14].

On resonance, atomic scattering factor can be strongly enhanced (up to orders of magnitude in the heavy elements [15]), resulting in selective contrast (in compounds) and promoting the appearance of forbidden reflections in the case of non-symmorphic Space Groups (SGs) [16-22]. Indeed, the resonant contribution originates from the outer electronic shells (less than spherically symmetric) and appears as tensorial properties of the scattering factor (Anisotropic Tensor of Susceptibility, ATS). In turn this can violate some of the SG extinction conditions (roto-translational symmetries [23]), resulting in an enhanced sensitivity to electronic details [2].

Historically, the RXD community was created with a strong contribution from neutron scattering people, converted to the newly available large scale X-ray facilities. At that time the spectroscopic information was less interesting (especially at the $\mathrm{K}$-edges in the hard regime) as compared to the advantages of X-rays in relation to diffraction, largely overwhelming some of the traditional limitations of neutron sources (for example flux, divergence, beam size). At the end of the 80's, working at a resonant edge was mainly exploited to enhance the atomic scattering factors, in the effort of making X-rays competitive with neutrons (for example in magnetic structure determination, with the additional benefit of a separate sensitivity to $\mathrm{L}$ and S components provided by the non-resonant magnetic scattering) [24-27].

Since then RXD investigations of spin structure, in particular and of EO in general, have grown and shown a flexible complementarity to neutrons not just when problems related to domains (sample size, confinement), safety (radioactive materials) and neutron specific limitations (isotopic cross sections) are present. On one hand beam size, energy and Q resolutions available with X-rays have resulted in domain mapping, sub-bands sensitivity, multi-K structure analysis [28-31], and the discovery of small incommensurabilities [32]. On the other hand, the use of electrons as scattering sources has provided direct access to sublattice/subbands EO information. The need for heavy modelling is then relaxed, resulting in magnetic structure 
determinations (almost) from scratch [33-39], as well as higher order multipole description and selective study of entangled electronic order parameters.

The sensitivity to peculiar electronic symmetry breaking tensorial moments is encoded in specific azimuth and polarization dependences of the diffracted beam and in the spectral line shape. Those dependences constitute the way to detect and attribute the nature of the resonant processes responsible for the measured RXD signal. In some cases, due to the subtlety of the effects investigated and the advanced tensorial analysis required, this process has been subject to controversial attributions for quite a while. After pioneering theoretical studies concomitant with the discovery of RXD (see citations in [1]), a number of authors has contributed to the development of the RXD formalism in various ways [40-51], effectively combining spectroscopic and diffractive information together.

In parallel, advanced analysis tools have been developed and dedicated courses and schools (Hercules, FDMNES school) created.

Concurrently, experimental investigations of electronic degrees of freedom have proved to be applicable to a number of EO problems, shining new light from a unique perspective. In the following we report some of them.

Models for charge ordering ( $\mathrm{CO}$ ) in solids are almost as old as magnetite, the prototypical material exhibiting this phenomenon. It is therefore not surprising that intensive study has focused on this material over the years. Due to the ferociously complex structure a comprehensive description of the system is still lacking, despite the improvements made to relate EO to the bulk properties [52-60]. Charge disproportionation is not restricted to iron oxides. Essentially all the transition metal (TM) ions [61-67] are affected in at least some of their compounds. Nowadays it is clear that the real valence of TM ions in crystals can be very different from the formal one, the effective $\mathrm{CO}$ value (disproportionation) ranging from a fraction of an electron, as in the case of magnetite, to full charge [73-76]. Speaking about classes of TM materials, surely manganites represent one of the favorite subjects of REXS investigation, due to the number of electronic properties shown across their complex phase diagram [68-72].

Among the electronic degrees of freedom, the Orbital Order (OO) is the specific issue pertinent to RXD. Traditionally, inferences on $\mathrm{OO}$ in solids were accessible through rules relating ligand distances with occupied orbitals. In the late 90's the discovery of resonant signals in manganites and vanadates [77-87] at the K-edge of TM oxides with suitable wave vectors set the community hope for a direct access to this elusive degree of freedom, crucially related to exchange interactions in crystals, for example.

Nowadays equivalent signals have been found in compounds across all the TM series and it is widely recognized that in most of the cases a strong dipole-dipole Jahn-Teller (JT) related signal dominates the K-edge resonance. Nevertheless the focus on $\mathrm{OO}$ paved the way for important advances in the understanding of EO in crystals. Indeed, depending on specific materials, sometimes a direct access to the $\mathrm{OO}$ is achievable, while in special cases investigation of the electron distribution in matter can be even addressed in terms of high order multipoles projections.

For the above reason, in the hard X-ray regime, K-edge investigations of exotic order in 3d TM ions have succeeded in a few cases, while $4 \mathrm{~d}$ and $\mathrm{f}$ electron based compounds have provided the most spectacular results [88-115].

With the progression of both theory [2] and experiments in the hard X-ray regime, advantages and complementarities peculiar to the soft X-rays became evident. L-edges, intrinsically richer from the spectroscopic point of view due to the spin-orbit coupling in the intermediate state, allow for a strong dipole-dipole transition to $3 \mathrm{~d}$ valence electrons in TM ions. So, in the soft regime a direct access to the electronic shell of interest in EO phenomena is possible in 3d TM compounds. This partially 
compensates for the technical difficulties and the intrinsic limitations imposed by the long wavelength. Moreover, the K-edge transition of light ligands can be exploited to access information on hybridization and local symmetry in essentially all compounds [116,117], similar to what was first observed in the hard regime [118-120].

For the above reasons a series of important contributions have been realized in the soft X-ray regime essentially in all EO domains [121-143], as proved by the number of diffractometers currently operating on dedicated beamlines around the world (SLS, ESRF, Diamond, NSLS, BessyII, Soleil, ...) and projects (ALBA, NSLS-II, Petra III, ...). On the contrary, few new multipurpose hard X-ray beamlines capable of performing RXD have appeared (Diamond, PetraIII), while an historical instrument, ID20 at the ESRF, was closed at the beginning of 2011.

Recently, the investigation of multifunctional (multiferroics, magnetoelectrics) materials has largely profited from the specificities of RXD, both in the soft and hard regime. Subtle information on symmetry, ligand and specific bands role provide important constraints to microscopic mechanisms, while order parameter interplay, magnetic structures and domain formation can be put in relation to bulk properties [144-168].

Traditionally, $\mathrm{Cu}$ based superconductors have been essentially studied by soft RXD (see specific citations in [121]) although with the advent of the pnictide superconductors, hard RXD investigations have been revitalized [169-172].

Most of the studied EO phenomena occur at low temperature on a static lattice. Nevertheless, RXD shows to be also sensitive to transient symmetry breaking due to atomic motion [173-175]. Over the last 10 years, the study of the so-called Thermal Motion Induced (TMI) forbidden reflections yielded valuable information on high temperature electronic states, atomic motion correlations [176,177], and phonon-electron coupling phenomena such as superconductivity.

In frontier experiments, the need for complex sample environments being able to cope with external fields, low temperatures and bulk measurements performed in-situ, has pushed for important improvements. Moderate pressure has been implemented [178-180] as well as pulsed magnetic fields [181]. Today, in both the soft and hard regimes, classical azimuth investigations are complemented by polarization scans [182-184], giving the possibility to attribute signals and to retrieve maximal information in spite of heavy sample environment constraints.

By using Phase Plates (PP) or exotic insertion devices, depending on the radiation energy, the beamline optical scheme and the degree of polarization purity needed, impinging beams of any desired polarization state obeying to electrodynamics rules can be produced with suitable intensity [185]. In particular, circular light has been exploited to investigate chirality in several compounds [186].

\section{Conclusions and future perspectives}

Several physics domains, cases and systems have profited from investigations by REXS at some stage, as extensively treated by other authors in this same special topic issue. In this paper a quick review of some relevant REXS investigations on EO in solids has been given. Although incomplete, it is hopefully sufficient to give a flavour of the information accessible in a vast series of frontier solid state physics problems.

The technical development has been enormous over the last years, involving many people all around the world. Many other developments and applications are currently under investigation and implementation: resonant powder diffraction is in its infancy $[187,188]$, while imaging via resonant magnetic contrast is experiencing a revival after important contributions [189-191]. Promising experiments relying on the difference between electronic and lattice time scales evolutions are upcoming, 
welcomed by the new generation of FEL sources. The very first outcome [191-193] already evidences how incomplete is our current understanding of elusive EO in matter. Hopefully, the close collaboration of our community with the coherent scattering and the pump and probe laser ones will stimulate the scientific investigation on EO as much as done in the past by the interaction with neutron diffraction and spectroscopy people.

In this respect, very recently the inelastic equivalent technique (RIXS) has shown important capabilities in EO investigation, especially in the soft regime due to astonishing technical improvements [194-197]. Normally, in RXD experiments the "elastic" rule compliance is left to Bragg's law, possibly complemented by a poor energy resolution analysis of the scattered beam to reject fluorescence and/or to analyze the scattered fluorescence. This is sufficient in the hard regime, where inelastic contributions are tiny. On the contrary, in the soft regime inelastic contamination can be severe and particular care should be taken when dealing with weak signals. In the near future, hopefully, RIXS and RXD may converge on dedicated experiments, showing how enhanced energy resolution analysis of the scattered beam might help in finding very weak elastic signals produced by faint, but crucial, EO phenomena in crystals.

The authors would like to acknowledge the REXS organizers for this opportunity to express their ideas. Many people have supported the realization of this short paper with useful discussions, suggestions, revisions. So, the authors would like to thank F. de Bergevin, G. Carbone, S. Collins, C. Detlefs, C.-H. Du, J. Geck, A.I. Goldman, D. Haskel, K. Ishii, N. Jaouen, Y. Joly, Y. Murakami, H. Suzuki and all the others who have supported our idea of producing this article in this form.

\section{References}

1. C. Vettier, Eur. Phys. J. Special Topics 208, 3 (2012)

2. Y. Joly, S. Di Matteo, Eur. Phys. J. Special Topics 208, 21 (2012)

3. L. Séve, et al., J. Magn. Magn. Mater. 148, 68 (1995)

4. M. Sacchi, et al., Phys. Rev. Lett. 81, 1521 (1998)

5. D. Haskel, et al., Phys. Rev. Lett. 87, 207201 (2001)

6. T. Kiyama, et al., J. Phys. Soc. Jpn. 72, 785 (2003)

7. Y. Wakabayashi, et al., J. Phys. Soc. Jpn. 77, 014712 (2008)

8. J.M. Tonnerre, et al., Phys. Rev. Lett. 100, 157202 (2008)

9. H.L. Meyerheim, et al., Phys. Rev. Lett. 103, 267202 (2009)

10. H. Nakao, et al., J. Phys. Soc. Jpn. 78, 024602 (2009)

11. E. Benckiser, et al., Nature Mat. Lett. 10, 189 (2011)

12. F. de Bergevin, M. Brunel, Phys. Lett. A 39, 141 (1972)

13. F. de Bergevin, M. Brunel, Acta Cryst. A 37, 314 (1981)

14. M. Brunel, F. de Bergevin, Acta Cryst. A 37, 324 (1981)

15. G. Lander, Eur. Phys. J. Special Topics 208, 129 (2012)

16. D.H. Templeton, L.K. Templeton, Acta Cryst. A 36, 237 (1980)

17. D.H. Templeton, L.K. Templeton, Acta Cryst. A 38, 62 (1982)

18. D.H. Templeton, L.K. Templeton, Acta Cryst. A 42, 478 (1986)

19. V.E. Dmitrienko, Acta Cryst. A 39, 29 (1983)

20. E.M. Ovchinnikova, V.E. Dmitrienko, Acta Cryst. A 56, 2 (1999)

21. V.E. Dmitrienko, et al., Acta Cryst. A 61, 481 (2005)

22. J. Kokubun, et al., Eur. Phys. J. Special Topics 208, 39 (2012)

23. International Tables for Crystallography (Springer, 2006)

24. L.K. Templeton, et al., Acta Cryst. A 38, 74 (1982)

25. K. Namikawa, et al., J. Phys. Soc. Jpn. 54, 4099 (1985)

26. D. Gibbs, et al., Phys. Rev. Lett. 61, 1241 (1988) 
27. K.D. Finkelstein, et al., Phys. Rev. Lett. 69, 1612 (1992)

28. P.S. Normile, et al., Phys. Rev. B 66, 014405 (2002)

29. M.J. Longfield, et al., Phys. Rev. B 66, 054417 (2002)

30. N. Bernhoeft, et al., Phys. Rev. B 69, 174415 (2004)

31. B. Detlefs, et al., Phys. Rev. B 75, 174403 (2007)

32. S. Agrestini, et al., Phys. Rev. B 77, 140403R (2008)

33. S. Ji, et al., Phys. Rev. Lett. 91, 257205 (2003)

34. A. Bombardi, et al., Phys. Rev. Lett. 93, 027202 (2004)

35. D. Haskel, et al., Phys. Rev. Lett. 95, 217207 (2005)

36. J. Fernandez-Rodriguez, et al., Phys. Rev. B 72, 052407 (2005)

37. V. Scagnoli, et al., Phys. Rev. B 73, 100409R (2006)

38. B. J. Kim, et al., Science 323, 1329 (2009)

39. B. Bohnenbuck, et al., Phys. Rev. Lett. 102, 037205 (2009)

40. M. Fabrizio, et al., Phys. Rev. Lett. 80, 3400 (1998)

41. P. Carra, et al., Phys. Rev. B 67, 045111 (2003)

42. S. Di Matteo, et al., Phys. Rev. Lett. 91, 257402 (2003)

43. I. Marri, P. Carra, Phys. Rev. B 69, 113101 (2004)

44. Y. Joly, et al., Phys. Rev. B 69, 224401 (2004)

45. S.W. Lovesey, et al., Phys. Rep. 411, 233 (2005)

46. N. Stojic, et al., Phys. Rev. B 72, 104108 (2005)

47. S. Di Matteo, et al., Phys. Rev. B 72, 144406 (2005)

48. S. Di Matteo, Y. Joly, Phys. Rev. B 74, 014403 (2006)

49. I. Marri, et al., Phys. Rev. B 74, 212410 (2006)

50. G. Subias, et al., Phys. Rev. B 75, 235101 (2007)

51. M.W. Haverkort, et al., Phys. Rev. B 82, 094403 (2010)

52. J. Garcia Ruiz, et al., Phys. Rev. Lett. 85, 578 (2000)

53. G. Subias, et al., Phys. Rev. Lett. 93, 156408 (2004)

54. D.J. Huang, et al., Phys. Rev. Lett. 96, 096401 (2006)

55. E. Nazarenko, et al., Phys. Rev. Lett. 97, 056403 (2006)

56. J. Schlappa, et al., Phys. Rev. Lett. 100, 026406 (2008)

57. E. Lorenzo, et al., Phs. Rev. Lett. 101, 226401 (2008)

58. Y. Joly, et al., Phys. Rev. B 78, 134110 (2008)

59. J. Garcia Ruiz, et al., Phys. Rev. Lett. 102, 176405 (2009)

60. S.B. Wilkins, et al., Phys. Rev. B 79, 201102R (2009)

61. H. Nakao, et al., Phys. Rev. Lett. 85, 4349 (2000)

62. U. Staub, et al., Phys. Rev. Lett. 88, 126402 (2002)

63. J. Geck, et al., Phys. Rev. B 66, 184407 (2002)

64. J. Herrero-Martin, et al., Phys. Rev. B 70, 024408 (2004)

65. J. Herrero-Martin, et al., Phys. Rev. B 79, 045121 (2009)

66. J. Herrero-Martin, et al., Phys. Rev. B 83, 184101 (2011)

67. G.L. Pascut, et al., Phys. Rev. Lett. 106, 157206 (2011)

68. Y. Endoh, et al., Phys. Rev. Lett. 82, 4328 (1999)

69. M.v. Zimmermann, et al., Phys. Rev. B 64, 195133 (2001)

70. S. Grenier, et al., Phys. Rev. B 69, 134419 (2004)

71. Y. Wakabayashi, et al., Phys. Rev. B 69, 144414 (2004)

72. J. Geck, et al., Phys. Rev. Lett. 95, 236401 (2005)

73. N. Ikeda, et al., Nature 436, 1136 (2005)

74. M. Angst, et al., Phys. Rev. Lett. 99, 086403 (2007)

75. A.M. Mulders, et al., Phys. Rev. Lett. 103, 077602 (2009)

76. S.R. Bland, et al., Phys. Rev. B 82, 115110 (2010)

77. D.H. Templeton, L.K. Templeton, Phys. Rev. B 49, 14850 (1994)

78. Y. Murakami, et al., Phys. Rev. Lett. 80, 1932 (1998)

79. Y. Murakami, et al., Phys. Rev. Lett. 81, 582 (1998)

80. L. Paolasini, et al., Phys. Rev. Lett. 82, 4719 (1999)

81. K. Nakamura, et al., Phys. Rev. B 60, 2425 (1999)

82. M.v. Zimmermann, et al., Phys. Rev. Lett. 83, 4872 (1999) 
83. M. Noguchi, et al., Phys. Rev. B 62, 9271R (2000)

84. L. Paolasini, et al., Phys. Rev. Lett. 88, 106303 (2002)

85. H. Nakao, et al., Phys. Rev. B 66, 184419 (2002)

86. Y. Murakami, et al., J. Magn. Magn. Mater. 310, 723 (2007)

87. R. Fukuta, et al., Phys. Rev. B 84, 140409R (2011)

88. D. Mannix, et al., Phys. Rev. B 60, 15187 (1999)

89. K. Hirota, et al., Phys. Rev. Lett. 84, 2706 (2000)

90. U. Staub, et al., Europhys. Lett. 53, 72 (2001)

91. H. Nakao, et al., J. Phys. Soc. Jpn. 70, 1857 (2001)

92. T. Matsumura, et al., Phys. Rev. B 65, 094420 (2002)

93. S.W. Lovesey, et al., Phys. Rev. B 65, 224402 (2002)

94. J. Igarashi, T. Nagao, J. Phys. Soc. Jpn. 71, 1771 (2002)

95. S.W. Lovesey, et al., J. Phys. Condens. Matter 15, 4511 (2003)

96. Y. Tanaka, et al., Phys. Rev. B 69, 024417 (2004)

97. D. Mannix, et al., Phys. Rev. Lett. 95, 117206 (2005)

98. I. Zegkinoglou, et al., Phys. Rev. Lett. 95, 136401 (2005)

99. T. Arima, et al., J. Phys. Soc. Jpn. 74, 1419 (2005)

100. D. Okuyama, et al., J. Phys. Soc. Jpn. 74, 2434 (2005)

101. H.C. Walker, et al., Phys. Rev. Lett. 97, 137203 (2006)

102. T. Nagao, J. Igarashi, Phys. Rev. B 74, 104404 (2006)

103. A.M. Mulders, et al., J. Phys. Condens. Matter 18, 11195 (2006)

104. S. Lovesey, et al., Phys. Rev. B 75, 054401 (2007)

105. T. Inami, et al., J. Magn. Magn. Mater. 310, 748 (2007)

106. A. Bombardi, et al., Phys. Rev. B 78, 100406 (2008)

107. H.C. Walker, et al., J. Phys. Condens. Matter 20, 395221 (2008)

108. D. Okuyama, et al., J. Phys. Soc. Jpn. 77, 044709 (2008)

109. T. Matsumura, et al., Phys. Rev. Lett. 103, 017203 (2009)

110. J. Fernandez-Rodriguez, et al., Phys. Rev. B 81, 085107 (2010)

111. T. Nagao, J. Igarashi, Phys. Rev. B 82, 024402 (2010)

112. U. Staub, et al., Phys. Rev. B 82, 104411 (2010)

113. H.C. Walker, et al., Phys. Rev. B 83, 193102 (2011)

114. H.C. Walker, et al., Phys. Rev. B 84, 235142 (2011)

115. A.J. Princep, et al., J. Phys. Condens. Matter 23, 266002 (2011)

116. S. Grenier, et al., Phys. Rev. Lett. 99, 206403 (2007)

117. M. Garcia-Fernandez, et al., Phys. Rev. Lett. 103, 097205 (2009)

118. D. Mannix, et al., Phys. Rev. Lett. 86, 4128 (2001)

119. K. Kutzushita, et al., Phys. Rev. B 73, 104431 (2006)

120. B. Detlefs, et al., Phys. Rev. B 77, 024425 (2008)

121. S.B. Wilkins, et al., Phys. Rev. Lett. 91, 167205 (2003)

122. P. Abbamonte, et al., Nature 431, 1078 (2004)

123. S.S. Dhesi, et al., Phys. Rev. Lett. 92, 056403 (2004)

124. K.J. Thomas, et al., Phys. Rev. Lett. 92, 237204 (2004)

125. P. Abbamonte, et al., Nat. Phys. 1, 155 (2005)

126. U. Staub, et al., Phys. Rev. B 71, 214421 (2005)

127. S.B. Wilkins, et al., Phys. Rev. B 71, 245102 (2005)

128. U. Staub, et al., Europhys. Lett. 76, 926 (2006)

129. J. Herrero-Martin, et al., Phys. Rev. B 73, 224407 (2006)

130. S. Grenier, et al., Phys. Rev. B 75, 085101 (2007)

131. T.A.W. Beale, et al., Phys. Rev. B 75, 174432 (2007)

132. M. Garcia-Fernandez, et al., Phys. Rev. B 78, 054424 (2008)

133. H. Wadati, et al., Appl. Phys. Lett. 106, 083705 (2009)

134. T.A.W. Beale, et al., Phys. Rev. B 79, 054433 (2009)

135. J. Fink, et al., Phys. Rev. B 79, 100502 (2009)

136. U. Staub, et al., Phys. Rev. B 79, 224419 (2009)

137. U. Staub, et al., Phys. Rev. B 80, 140410 (2009) 
138. J. Okamoto, et al., Phys. Rev. B 82, 132402 (2010)

139. M. Garcia-Fernandez, et al., Phys. Rev. B 82, 235108 (2010)

140. V. Scagnoli, et al., Science 332, 696 (2011)

141. J. Fink, et al., Phys. Rev. B 83, 092503 (2011)

142. D.G. Hawthorn, et al., Phys. Rev. B 84, 075125 (2011)

143. S.B. Wilkins, et al., Phys. Rev. B 84, 195101 (2011)

144. R. Feyerherm, et al., Phys. Rev. B 73, 180401 (2006)

145. C.-H. Jang, et al., Phys. Rev. B 73, 224112 (2006)

146. O. Prokhnenko, et al., Phys. Rev. Lett. 98, 057206 (2007)

147. J. Okamoto, et al., Phys. Rev. Lett. 98, 157202 (2007)

148. J. Koo, et al., Phys. Rev. Lett. 99, 197601 (2007)

149. D. Mannix, et al., Phys. Rev. B 76, 184420 (2007)

150. Y. Bondenthin, et al., Phys. Rev. Lett. 100, 027201 (2008)

151. S. Nandi, et al., Phys. Rev. Lett. 100, 217201 (2008)

152. A. Rusydi, et al., Appl. Phys. Lett. 92, 262506 (2008)

153. R.A. Ewings, et al., Phys. Rev. B 77, 104415 (2008)

154. G. Beutier, et al., Phys. Rev. B 77, 172408 (2008)

155. J. Strempfer, et al., Phys. Rev. B 78, 024429 (2008)

156. S.B. Wilkins, et al., Phys. Rev. Lett. 103, 207602 (2009)

157. M. Matsubara, et al., Phys. Rev. B 79, 180407R (2009)

158. H.C. Walker, et al., Physica B Cond. Matt. 404, 3264 (2009)

159. T.A.W. Beale, et al., Phys. Rev. Lett. 105, 087203 (2010)

160. E. Schierle, et al., Phys. Rev. Lett. 105, 167207 (2010)

161. C. Azimonte, et al., Phys. Rev. B 81, 012103 (2010)

162. A.M. Mulders, et al., Phys. Rev. B 81, 092405 (2010)

163. U. Staub, et al., Phys. Rev. B 81, 144401 (2010)

164. D.-J. Huang, et al., J. Phys. Soc. Jpn. 79, 011009 (2010)

165. R.D. Johnson, et al., Phys. Rev. B 83, 054438 (2011)

166. S. Partzsch, et al., Phys. Rev. Lett. 107, 057201 (2011)

167. R.A. de Souza, et al., Phys. Rev. B 84, 104416 (2011)

168. H. Wadati, et al., Phys. Rev. Lett. 108, 047203 (2012)

169. J. Herrero-Martin, et al., Phys. Rev. B 80, 134411 (2009)

170. M.G. Kim, et al., Phys. Rev. B 82, 180412 (2010)

171. M.G. Kim, et al., Phys. Rev. B 83, 134522 (2011)

172. S. Nandi, et al., Phys. Rev. B 84, 054419 (2011)

173. V.E. Dmitrienko, et al., JETP Letters 69, 938 (1999)

174. J. Kokubun, et al., Phys. Rev. B 64, 073203 (2001)

175. S.P. Collins, et al., Phys. Rev. B 68, 064110 (2003)

176. A. Kirfel, et al., Phys. Rev. B 66, 165202 (2002)

177. E.N. Ovchinnikova, et al., J. Phys.: Condens. Matter 22, 355404 (2010)

178. P. Deen, et al., Phys. Rev. B 71, 245118 (2005)

179. K. Ohwada, et al., Phys. Rev. B 72, 014123 (2005)

180. K. Ohwada, et al., Phys. Rev. B 76, 094113 (2007)

181. T. Inami, et al., J. Phys. Soc. Jpn. 78, 033707 (2009)

182. C. Mazzoli, et al., Phys. Rev. B 76, 195118 (2007)

183. R.D. Johnson, et al., Phys. Rev. B 78, 104407 (2008)

184. V. Scagnoli, et al., J. Synch. Rad. 16, 778 (2009)

185. C. Detlefs, et al., Eur. Phys. J. Special Topics 208, 359 (2012)

186. Y. Tanaka, S.W. Lovesey, Eur. Phys. J. Special Topics 208, 67 (2012)

187. U. Staub, et al., J. Phys.: Condens. Matter 19, 092201 (2007)

188. M. Garcia-Fernandez, et al., Phys. Rev. B 77, 060402R (2008)

189. C.S. Nelson, et al., Phys. Rev. B 66, 134412 (2002)

190. J.J. Turner, et al., New J. Phys. 10, 053023 (2008)

191. S. Konings, et al., Phys. Rev. Lett. 106, 077402 (2011)

192. C. Gutt, et al., Phys. Rev. B 81, 100401 (2010) 
193. H. Ehrke, et al., Phys. Rev. Lett. 106, 217401 (2011)

194. N. Pontius, et al., Appl. Phys. Lett. 98, 182504 (2011)

195. G. Ghiringhelli, et al., Phys. Rev. Lett. 102, 027401 (2009)

196. L. Braicovich, et al., Phys. Rev. Lett. 104, 077002 (2010)

197. M. Le Tacon, et al., Nat. Phys. 7, 725 (2011)

198. M. Moretti Sala, et al., New J. Phys. 13, 043026 (2011) 